\title{
GENERALIZED SEMI-FREDHOLM TRANSFORMATIONS
}

\author{
D. G. TACON \\ (Received 15 June 1981, revised 18 January 1982) \\ Communicated by J. B. Miller
}

\begin{abstract}
The class $\phi_{+}(X, Y)$ of semi-Fredholm transformations consists of those transformations $T: X \rightarrow Y$ for which $\alpha(T)=\operatorname{dim} \operatorname{ker} T<\infty$ and for which $T(X)$ is closed. It forms an open subset of $(B(X, Y)$ closed under perturbation by compact transformations and is a particularly important class of transformation since $T$ is Fredholm if and only if $T \in \phi_{+}(X, Y)$ and $T^{\prime} \in \phi_{+}\left(Y^{\prime}, X^{\prime}\right)$. The realization that elements of $\phi_{+}(X, Y)$ have very simple nonstandard characterizations lead the author to consider the possibility of finding an analogous open class of transformation which is closed under perturbation by weakly compact transformations. Consequently this paper investigates two related classes which contain $\phi_{+}(X, Y)$. The first such class coincides with the class of Tauberian transformations whilst the second consists of those transformations which have Tauberian extensions on the nonstandard hulls. The Tauberian transformations are closed under perturbation by weakly compact transformations but in general are not open. The "super" Tauberian transformations are closed under perturbation by super weakly compact transformations and in fact form an open subset of $\operatorname{si}(X, Y)$.
\end{abstract}

1980 Mathematics subject classification (Amer. Math. Soc.): 47 A 53.

\section{Preliminaries}

In the following we assume for definiteness that all spaces are real infinite dimensional Banach spaces and that they are embedded in a set theoretical structure $\Re$ of which $* \mathscr{T}$ is an $\boldsymbol{N}_{1}$-saturated enlargement. If $X$ is such a Banach space the nonstandard hull $\hat{X}$ of $X$ with respect to $* \Re$ is constructed by factoring the infinitesimal elements of $* X$ from the finite elements of $* X$. The original space $X$ is embedded in $\hat{X}$ and $\hat{X}$ is a Banach space under the norm $\|\hat{p}\|=$ standard part ${ }^{*}\|p\|$. Here $p \in$ finite ${ }^{*} X$ and $\hat{p}$ denotes the equivalence class determined by $p$. We refer the reader to Luxemburg [8] for a proof of the latter result and for a

c Copyright Australian Mathematical Society 1983 
complete discussion of the nonstandard hull construction. If we have two spaces $X$ and $Y$ we let $\mathscr{B}(X, Y)$ denote the space of bounded linear transformations from $X$ into $Y$ equipped with the supremum norm. Our work depends on the fact that an element $S \in$ finite $* \mathscr{B}(X, Y)$ defines an element $\hat{S} \in \mathscr{G}(\hat{X}, \hat{Y})$ by the equation $\hat{S}(\hat{p})=(S(p))$. Finally we let $X^{\prime}$ denote the dual space of $X ; X_{\delta}$ the closed ball of radius $\delta ; \bar{A}$ the closure of $A ; \mathcal{K}(X, Y)$ the space of compact transformations from $X$ into $Y$ and $\mathscr{G} \mathscr{K}(X, Y)$ the space of weakly compact transformations from $X$ into $Y$.

\section{Semi-Fredholm transformations}

Our first result provides a basic characterization of elements of $\phi_{+}(X, Y)$ and motivates much of what follows.

THEOREM 1. Let $X$ and $Y$ be Banach spaces and suppose $T \in \mathscr{B}(X, Y)$. Then the following conditions are equivalent:

(i) $T \in \phi_{+}(X, Y)$;

(ii) $\hat{T}$ maps $\hat{X} \backslash X$ into $\hat{Y} \backslash Y$, that is, $T$ maps finite non near-standard points of ${ }^{*} X$ into non near-standard points of ${ }^{*} Y$;

(iii) $T$ does not possess a singular sequence, that is, a bounded sequence $\left\{x_{n}\right\}$ of $X$ with no convergent subsequence such that $T x_{n} \rightarrow 0$ as $n \rightarrow \infty$;

(iv) $\operatorname{ker} \hat{T} \subset X$;

(v) $\alpha(\hat{T})=\operatorname{dim}(\operatorname{ker} \hat{T})<\infty$.

Proof. We establish the chain of implications (i) $\Rightarrow$ (ii) $\Rightarrow$ (iii) $\Rightarrow$ (iv) $\Rightarrow$ (v) $\Rightarrow$ (i).

(i) implies (ii). Suppose $T p \simeq y$ where $p \in$ fin $^{*} X$ and $y \in Y$. Then $y \in \overline{T(X)}$ and so, by assumption, there is a point $x \in X$ such that $T x=y$. We then have $\hat{T}(\hat{p})=\hat{y}=\hat{T}(\hat{x})$ so that $\hat{p}-\hat{x} \in \operatorname{ker} \hat{T}$. But if $\operatorname{ker} T=\operatorname{span}\left\{x_{1}, x_{2}, \ldots, x_{n}\right\}$ and $T$ has closed range then ker $\hat{T}=\operatorname{span}\left\{\hat{x}_{1}, \hat{x}_{2}, \ldots, \hat{x}_{n}\right\}$ by an earlier result of ours; see [11, Theorem 3]. Consequently $p=\hat{x}+\alpha_{1} \hat{x}_{1}+\cdots+\alpha_{n} \hat{x}_{n}$ where $\alpha_{k} \in \mathbf{R}$ for $k=1, \ldots, n$ which implies $p$ is near-standard.

(ii) implies (iii). Suppose $T$ possesses a singular sequence $\left\{x_{n}\right\}$. Then for any $\omega \in{ }^{*} \mathbf{N} \backslash \mathbf{N} T x_{\omega} \simeq 0$ whilst $x_{\omega}$ is finite and non near-standard.

(iii) implies (iv). Suppose $\operatorname{ker} \hat{T} \not \subset X$. Then there exists a non near-standard point $p$ of norm 1 such that $T p \simeq 0$. As $X$ is complete the non near-standard assumption on $p$ implies there is a (standard) $\varepsilon>0$ such that $\|p-x\|>\varepsilon$ whenever $x \in X$. This allows us to construct a singular sequence $\left\{x_{n}\right\}$ for $T$, the sequence being defined recursively in the following way. Choose $x_{1}$ arbitrarily 
with norm 1 and then, having chosen $x_{1}, \ldots, x_{k-1}$, choose $x_{k}$ to satisfy the sentence

$$
\text { " } \exists x\left(\|x\|=1 \&\left\|x-x_{i}\right\|>\varepsilon, i=1, \ldots, k-1 \&\|T x\| \leqslant \frac{1}{k}\right) . "
$$

Such a point $x_{k}$ exists since $p$ satisfies the sentence in ${ }^{*} X$.

(iv) implies (v). Suppose $\alpha(\hat{T})=\infty$. Then since $\operatorname{ker} \hat{T} \subset X$ it follows that $\alpha(T)=\infty$. Consequently there is a finite non-standard point $p \in \operatorname{ker}^{*} T$, contradicting the initial assumption that $\operatorname{ker} \hat{T} \subset X$.

(v) implies (i). Since $\operatorname{ker} T \subset \operatorname{ker} \hat{T}$ it follows that $\alpha(T)<\infty$. It again follows by [11, Theorem 3] that $T(X)$ is closed provided we can show $\hat{T}(\hat{X})$ is. Choose a basis $\left\{x_{1}, \ldots, x_{j}\right\}$ for $\operatorname{ker} T$ and extend to obtain a basis $\left\{\hat{x}_{1}, \ldots, \hat{x}_{j}, \hat{p}_{1}, \ldots, \hat{p}_{k}\right\}$ for ker $\hat{T}$. Let ${ }^{*} X=\operatorname{sp}\left\{{ }^{*} x_{1}, \ldots,{ }^{*} x_{j}\right\} \oplus \operatorname{sp}\left\{p_{1}, \ldots, p_{k}\right\} \oplus Q=K \oplus P \oplus Q$, choosing $Q$ so that the projections are finite. Then $\hat{X}=\hat{K} \oplus \hat{P} \oplus \hat{Q}, \hat{T}(\hat{X})=\hat{T}(\hat{Q})$ and $\hat{T}$ restricted to $\hat{Q}$ is $1: 1$. Suppose $\hat{T}(\hat{Q})$ is not closed. Then for all (standard) $\delta>0$ there is a point $q \in Q$ with $\|q\|=1$ such that $\|T(q)\| \leqslant \delta$. Thus the set $\left\{\delta \in{ }^{*} \mathbf{R} \mid \delta>0 \& \exists q \in Q(\|q\|=1 \&\|T q\| \leqslant \delta)\right\}$ is an internal subset of $* \mathbf{R}$ containing all the positive standard real numbers. Consequently it contains a positive infinitesimal and so $\hat{Q}$ contains a nonzero element in $\operatorname{ker} \hat{T}$. This is a contradiction.

Corollary. Let $T \in \phi_{+}(X, Y)$ and $K \in \mathcal{K}(X, Y)$. Then $T+K \in \phi_{+}(X, Y)$.

Proof. If $K$ is compact then $\hat{K}$ maps $\hat{X}$ into $Y$ by a well-known result of Robinson [10, page 119]. Thus the corollary follows from Theorem 1 (ii).

We gave a nonstandard proof that $\phi_{+}(X, Y)$ is open in [11, Corollary 3]. In fact the methods given there can be modified to show that $T$ has closed range if and only if $\hat{T}$ has. Indeed, that $\hat{T}(\hat{X})$ is closed if $T(X)$ is, is shown in Theorem 3 (i) (a) of [11]. The converse implication for standard $T$ follows essentially as in Theorem 3 (ii) (b). As the alteration is quite straightforward and as the result is not used in this paper we do not make it explicit here. We also note that Chadwick and Wickstead [2] have considered semi-Fredholm operators in an ultrapower setting and that they have obtained a number of interesting related results.

\section{Tauberian transformations}

From the nonstandard viewpoint it is natural to think in terms of relaxing one of the equivalent conditions (ii), (iv), (v) in Theorem 1. Our initial thought was to relax condition (ii) by requiring that $T$ map finite non weak near-standard points 
of ${ }^{*} X$ into non weak near-standard (or even non near-standard) points of ${ }^{*} Y$. Here weak near-standard means near-standard in the weak topology. In fact either of these conditions is equivalent to the requirement that $T^{\prime \prime} x^{\prime \prime} \in Y$ implies $x^{\prime \prime} \in X$. Such transformations, called Tauberian transformations, have been studied by Kalton and Wilansky [6]. Our next result proves the equivalence of these conditions; the equivalence of the standard conditions listed here is established in [6]. We find it convenient to let $\mathscr{T}(X, Y)$ denote the set of Tauberian transformations from $X$ into $Y$.

Theorem 2. Let $X$ and $Y$ be Banach spaces and suppose $T \in \mathscr{B}(X, Y)$. Then the following conditions are equivalent:

(i) $T \in \mathcal{T}(X, Y)$;

(ii) $T$ maps finite non weak near-standard points of $* X$ into non weak near-standard points of $* Y$;

(iii) a) $T\left(X_{1}\right)$ is closed in $Y$,

b) $\operatorname{ker} T^{\prime \prime} \subseteq X$;

(iv) $T$ maps finite non weak near-standard points of ${ }^{*} X$ into non near-standard points of ${ }^{*} Y$ :

(v) a) $T\left(X_{1}\right)$ is closed in $Y$,

b) If $\left\{x_{n}\right\}$ is a bounded sequence of points in $X$ such that $\left\{T x_{n}\right\}$ is convergent to 0 in $Y$ then $\left\{x_{n}\right\}$ has a weakly convergent subsequence;

(vi) the only bounded sets which map into compact sets are those whose weak closures are weakly compact;

(vii) the only bounded sets which map into weakly compact sets are those whose weak closures are weakly compact.

Proof. The equivalence of (i), (vi) and (vii) has been established by Kalton and Wilansky [6, Theorem 3.2]. Therefore it is enough to prove the chain of implications (i) $\Rightarrow$ (ii) $\Rightarrow$ (iii) $\Rightarrow$ (iv) $\Rightarrow$ (v) $\Rightarrow$ (vi).

(i) implies (ii). Assume (i) and suppose $p$ is a finite non weak near-standard point of ${ }^{*} X$. Define $x^{\prime \prime} \in X^{\prime \prime}$ by $x^{\prime \prime}(f)=$ standard part $f(p)$ for $f \in X^{\prime}$. Then $x^{\prime \prime} \notin X$ since $p$ is not weak near-standard. Consequently $T^{\prime \prime} x^{\prime \prime} \notin Y$ which means $T p$ is not weak near-standard since

$$
g(T p)=\left(T^{\prime} g\right) p \simeq x^{\prime \prime}\left(T^{\prime} g\right)=\left(T^{\prime \prime} x^{\prime \prime}\right) g \text { for all } g \in Y^{\prime} .
$$

(ii) implies (iii). Assume (ii) and suppose $y$ belongs to the closure of $T\left(X_{1}\right)$. Then there exists a point $p \in{ }^{*} X_{1}$ such that $T p \simeq y$. Thus $T p$ is near-standard and so $p$ is weak near-standard. If $p$ has weak standard part $x$ then $T x=y$ and so $T\left(X_{1}\right)$ is closed. Next suppose $T^{\prime \prime} x^{\prime \prime}=0$, and let $p$ be chosen in finite * $X$ so that $x^{\prime \prime}(f)=f(p)$ whenever $f \in X^{\prime}$ (see Luxemberg [9]). It follows that $T p$ is weak 
near-standard to 0 , and consequently $p$ is weak near-standard. If $p$ has weak standard part $x$ then $x^{\prime \prime}=x$ and so $\operatorname{ker} T^{\prime \prime} \subseteq X$.

(iii) implies (iv). Assume (iii) and suppose $p$ belongs to finite ${ }^{*} X$ and that $T p$ has standard part $y$. If $\|p\| \leqslant \delta$ then $y$ belongs to the closure of $T\left(X_{\delta}\right)$ and so $y=T x$ for some $x$ with $\|x\| \leqslant \delta$. Let $q=p-x$ so that $T q$ has standard part 0 . Define $x^{\prime \prime} \in X^{\prime \prime}$ by $x^{\prime \prime}(f)=$ standard part $f(q)$ for $f \in X^{\prime}$. Then $T^{\prime \prime} x^{\prime \prime}=0$ and so $x^{\prime \prime} \in X$. This means $q$ is weak near-standard and thus that $p$ is too.

(iv) implies (v). The closure of $T\left(X_{1}\right)$ follows by a similar argument to that used in the proof of (ii) implies (iii). Now suppose (v)b) does not hold. Then by a well-known result of James [4, Theorem 3] we can assume we have sequences $\left\{x_{n}\right\}$ and $\left\{f_{n}\right\}$ of norm one elements in $X$ and $X^{\prime}$ respectively together with a positive real $r$ such that

$$
f_{n}\left(x_{k}\right)>r \quad \text { for } n \leqslant k, \quad f_{n}\left(x_{k}\right)=0 \quad \text { for } n>k
$$

with the property that $\left\{T x_{n}\right\}$ is a convergent to 0 . Then for $\omega \in{ }^{*} \mathbf{N} \backslash \mathbf{N}$ we have $T x_{\omega}$ near-standard whilst, as we now show, $x_{\omega}$ fails to be weak near-standard. To see this use the fact that the sequence $\left\{f_{n}\right\}$ has a weak* limit point $f$. For this functional we have $f\left(x_{k}\right)=0$ for $k=1,2,3, \ldots$ so that $f\left(x_{\omega}\right)=0$. Thus if $x_{\omega}$ has weak standard part $x$ we must have $f(x)=0$. On the other hand $f_{n}\left(x_{\omega}\right)>r$ for $n=1,2,3, \ldots, \omega$ so that $f_{n}(x) \geqslant r$ for $n=1,2,3, \ldots$, whence $f(x) \geqslant r$, a contradiction.

(v) implies (vi). Suppose $B$ is a subset of the closed ball $X_{\delta}$ with the property that $T(B)$ is relatively compact. If $\left\{x_{n}\right\}$ is a sequence of points from $B$ the sequence $\left\{T x_{n}\right\}$ has a convergent subsequence. Denote this subsequence by $\left\{T z_{n}\right\}$ and suppose $T z_{n} \rightarrow y$. Then $y$ belongs to the closure of $T\left(X_{\delta}\right)$ and so, by condition (a), there is a point $x \in X_{\delta}$ such that $T x=y$. The sequence $\left\{T\left(z_{n}-x\right)\right\}$ is therefore convergent to 0 and so, by (b), $\left\{z_{n}-x\right\}$ has a weakly convergent subsequence. Thus $\left\{x_{n}-x\right\}$ and $\left\{x_{n}\right\}$ have weakly convergent subsequences and it follows that $B$ is relatively weakly compact by the Eberlein-Smulian theorem.

We now list a number of properties of Tauberian transformations, the first three of which have been noted by Kalton and Wilansky.

(1) $\mathscr{T}(X, Y)$ is closed under perturbation by weakly compact transformations. Therefore operators of the form $W+\lambda I$ are Tauberian whenever $W \in$ Q.15 $\mathcal{F}(X, Y)$ and $\lambda$ is a real number.

(2) $\widetilde{J}(X, Y)$ equals $\mathscr{B}(X, Y)$ if $X$ is reflexive. If $X$ is non-reflexive then no weakly compact transformation can be Tauberian and so if $h(X, Y)=$ Qf $\mathscr{F}(X, Y)$ then $\mathscr{T}(X, Y)$ is empty.

(3) If $T \in \mathcal{T}(X, Y)$ then $\operatorname{ker} T$ is reflexive. However $T$ needn't be Tauberian even if $T$ is $1: 1$ and $T\left(X_{1}\right)$ is closed for consider the compact operator $T: l^{1} \rightarrow l^{1}$ 
defined by $T:\left\{x_{k}\right\} \rightarrow\left\{x_{k} / k\right\}$. For this operator the kernel is trivial whilst $T\left(X_{1}\right)$ is closed. However, for $\omega \in{ }^{*} \mathbf{N}-\mathbf{N}, \delta_{\omega}$ fails to be weak near-standard even though $T \delta_{\omega} \simeq 0$. Here $\delta_{n}$ denotes the $n$th natural basis vector.

(4) If $T \in \mathscr{B}(X, Y), S \in \Re(Y, Z)$ and $S T \in \mathcal{T}(X, Z)$ then $T \in \mathcal{T}(X, Y)$. This follows since $S$ is continuous from $Y$ with the weak topology to $Z$ with the weak topology. Thus if $T \in \mathscr{B}(X, Y)$ and there is an element $S \in \mathscr{B}(Y, X)$ such that $S T=I+W$ where $W \in \mathscr{W} \mathcal{K}(X, X)$ then $T$ is Tauberian. It is a consequence of (5) that the set of $T$ satisfying this condition is, in general, properly contained in $\mathscr{T}(X, X)$.

(5) $\mathscr{T}(X, Y)$ need not be open in $\mathscr{\Re}(X, Y)$. For $n=1,2,3, \ldots$ suppose we have an irreflexive Banach space $X_{n}$ and operators $T_{n}, S_{n}$ on $X_{n}$, where the norms $\left\|T_{n}\right\|$ and $\left\|S_{n}\right\|$ are uniformly bounded, with the properties that $T_{n} \in \phi_{+}\left(X_{n}, Y_{n}\right)$ and $\alpha\left(T_{n}+S_{n} / n\right)=\infty$. Let $X$ be that subspace of the product space $\pi X_{n}$ consisting of those elements $x=\left\{x_{k}\right\}$ for which $\|x\|^{2}=\Sigma\left\|x_{k}\right\|^{2}<\infty$ and define operators $T$ and $S$ on $X$ by $T:\left\{x_{k}\right\} \rightarrow\left\{T_{k} x_{k}\right\}$ and $S:\left\{x_{k}\right\} \rightarrow\left\{S_{k} x_{k}\right\}$. It follows that $T$ is Tauberian but that, for $n=1,2,3, \ldots, T+S / n$ is not Tauberian. The second statement follows since the kernel of $T_{n}+S_{n} / n$ is not reflexive so that neither is that of $T+S / n$. This gives the result we want provided we can construct the $T_{n}$ and $S_{n}$. One simple way of doing this is to let $Y=Z=c_{0}$ and then set $X_{n}=Y \oplus Z$. For each $n$ let $S_{n}(x)=-x$ and define $T_{n}$ by linear extension setting $T_{n} y=y / n$ when $y \in Y$ and $T_{n} z=z$ when $z \in Z$.

We comment that since $\mathscr{W} \mathscr{K}(X, X)$ is a closed ideal in $\mathscr{B}(X, X)$ the quotient space $\mathscr{B}(X, X) / \mathscr{W} \mathscr{K}(X, X)$ is a Banach algebra with identity. Accordingly, see Caradus, Pfaffenberger and Yood [1, page 22], the set of left regular elements in this algebra is open, and so the set of $T \in \mathscr{B}(X, X)$ such that there is an $S \subset \mathscr{B}(X, X)$ and $W \in \mathscr{W} \mathcal{K}(X, X)$ with $S T=I+W$ is open. Hence the above example also shows that this class of operator is in general properly contained in $\mathfrak{T}(X, X)$.

(6) Let $\mathscr{D} \mathcal{T}(X, Y)$ denote the set of $T \in \mathscr{B}(X, Y)$ for which $Y^{\prime \prime}$ equals the closure of $Y+T^{\prime \prime}\left(X^{\prime \prime}\right)$. The classes $\mathscr{T}(X, Y)$ and $\mathscr{D} \mathscr{T}(X, Y)$ have a dual relationship with one another.

Proposition. Let $X$ and $Y$ be Banach spaces and suppose $T \in \mathscr{B}(X, Y)$. Then:

(i) If $T \in \mathscr{O} \mathcal{T}(X, Y)$ then $T^{\prime} \in \mathcal{T}\left(Y^{\prime}, X^{\prime}\right)$;

(ii) If $T^{\prime} \in \mathcal{T}\left(Y^{\prime}, X^{\prime}\right)$ then $T \in \mathscr{Q} \mathcal{G}(X, Y)$;

(iii) If $T^{\prime} \in \mathscr{D} \mathscr{T}\left(Y^{\prime}, X^{\prime}\right)$ then $T \in \mathscr{T}(X, Y)$.

Proof. (i) Suppose $T^{\prime \prime \prime} \phi \in X^{\prime}$ where $\phi \in Y^{\prime \prime \prime}$. Then $\phi$ restricted to $Y$ defines an element $g \in Y^{\prime}$ and we show $\phi$ is in fact $g$. This just means $\phi\left(y^{\prime \prime}\right)=y^{\prime \prime}(g)$ for all $y^{\prime \prime} \in Y^{\prime \prime}$ and so, given the initial assumption, its enough to check the equality 
for $y^{\prime \prime} \in T^{\prime \prime}\left(X^{\prime \prime}\right)$. First note that since $T^{\prime \prime \prime} \phi \in X^{\prime}, T^{\prime \prime \prime} \phi=T^{\prime} g$ because, for $x \in X$,

$$
\left(T^{\prime \prime \prime} \phi\right)(x)=\phi\left(T^{\prime \prime} x\right)=\phi(T x)=g(T x)=\left(T^{\prime} g\right)(x) .
$$

Thus for $x^{\prime \prime} \in X^{\prime \prime}$ we have

$$
\phi\left(T^{\prime \prime} x^{\prime \prime}\right)=\left(T^{\prime \prime \prime} \phi\right)\left(x^{\prime \prime}\right)=x^{\prime \prime}\left(T^{\prime \prime \prime} \phi\right)=x^{\prime \prime}\left(T^{\prime} g\right)=\left(T^{\prime \prime} x^{\prime \prime}\right)(g) .
$$

(ii) Suppose $T \notin \mathscr{D} \mathcal{T}(X, Y)$ where $T^{\prime} \in \mathcal{T}\left(Y^{\prime}, X^{\prime}\right)$. Then there exists a non-zero $\phi \in Y^{\prime \prime}$ such that $\phi\left(Y+T^{\prime \prime}\left(X^{\prime \prime}\right)\right)=0$. In particular $\left(T^{\prime \prime \prime} \phi\right)\left(X^{\prime \prime}\right)=0$ so $T^{\prime \prime \prime} \phi=0$, so that $\phi \in Y^{\prime}$. But $\phi(Y)=0$ so $\phi=0$ contradicting the initial assumption on $\phi$.

(iii) If $T^{\prime} \in \mathscr{Q} \mathcal{T}\left(Y^{\prime}, X^{\prime}\right)$ then $T^{\prime \prime} \in \mathcal{T}\left(X^{\prime \prime}, Y^{\prime \prime}\right)$. This implies $T \in \mathcal{T}(X, Y)$ as is easily seen by checking condition (iii) of Theorem 2 .

We comment that we have been unable to determine whether $T \in \mathcal{T}(X, Y)$ implies $T^{\prime} \in \mathscr{D} \mathcal{T}\left(Y^{\prime}, X^{\prime}\right)$, or equivalently whether $T \in \mathcal{T}(X, Y)$ implies $T^{\prime \prime} \in$ $\mathscr{T}\left(X^{\prime \prime}, Y^{\prime \prime}\right)$. It is an open question in Kalton and Wilansky to decide the truth of the latter implication.

\section{Super Tauberian transformations}

Lebow and Schechter [7] show that the perturbation class of an open semigroup in a Banach algebra forms a closed two sided ideal. It is for this sort of reason that we are particularly interested in open subsets of $\mathscr{B}(X, Y)$. We consider such a subset contained in $\mathscr{J}(X, Y)$. We define this second class $\psi(X, Y)$ by the property that $T \in \psi(X, Y)$ if for all real numbers $r$ satisfying $0<r<1$ there exists a positive integer $n$ for which there do not exist finite sequences of norm one elements $\left\{x_{1}, x_{2}, \ldots, x_{n}\right\}$ in $X$ and $\left\{f_{1}, f_{2}, \ldots, f_{n}\right\}$ in $X^{\prime}$ for which $f_{j}\left(x_{i}\right)>r$ for $1 \leqslant j \leqslant i \leqslant n$, $f_{j}\left(x_{i}\right)=0$ for $1 \leqslant i<j \leqslant n$ and $\left\|T x_{k}\right\|<1 / k$ for $k=1,2, \ldots, n$. We shall need use the fact that the phrase "for all real numbers $r$ " may be replaced by the phrase "for some real number $r$ " and for the purpose of showing this we find it convenient to let $W \psi(X, Y)$ denote the class of transformations satisfying this formally weaker condition. We also remind the reader that the space $X$ is superreflexive if any space finitely representable in $X$ is reflexive. However for clarity we note the characterizations of superreflexivity we use. A Banach space $X$ is superreflexive if for all $r$ satisfying $0<r<1$ (equivalently, for some $r$ satisfying $0<r<1)$ there is a positive integer $n$ for which there do not exist finite sequences of norm one elements $\left\{x_{1}, x_{2}, \ldots, x_{n}\right\}$ in $X$ and $\left\{f_{1}, f_{2}, \ldots, f_{n}\right\}$ in $X^{\prime}$ satisfying $f_{j}\left(x_{i}\right)>r$ for $1 \leqslant j \leqslant i \leqslant n$ and $f_{j}\left(x_{i}\right)=0$ for $1 \leqslant i<j \leqslant n$. These equivalences were established by James [5], but see also Henson and Moore [3, Theorem 8.5], in which they show $X$ is superreflexive if and only if $\hat{X}$ is reflexive. 
THeOREM 3. Let $X$ and $Y$ be Banach spaces and let $T \in \mathscr{B}(X, Y)$. Then the following conditions are equivalent:

(i) $T \in \psi(X, Y)$;

(ii) $\hat{T} \in \psi(\hat{X}, \hat{Y})$;

(iii) $\hat{T} \in \mathcal{G}(\hat{X}, \hat{Y})$;

(iv) $T \in W \psi(X, Y)$;

(v) ker $\hat{T}$ is superreflexive;

(vi) ker $\hat{T}$ is reflexive.

Proof. We show (i) $\Rightarrow$ (ii) $\Rightarrow$ (iii) $\Rightarrow$ (iv) $\Rightarrow$ (v) $\Rightarrow$ (vi) $\Rightarrow$ (i).

(i) implies (ii). Suppose there is a real number $r$ with $0<r<1$ such that for all positive integers $n$ there exist finite sequences of norm one elements $\left\{\hat{p}_{1}, \hat{p}_{2}, \ldots, \hat{p}_{n}\right\}$ in $\hat{X},\left\{\phi_{1}, \phi_{2}, \ldots, \phi_{n}\right\}$ in $\hat{X}^{\prime}$ satisfying the conditions that $\phi_{j}\left(\hat{p}_{i}\right)>r$ for $1 \leqslant j \leqslant i \leqslant n, \phi_{j}\left(\hat{p}_{i}\right)=0$ for $1 \leqslant i<j \leqslant n$, and $\left\|T\left(p_{k}\right)\right\|<1 / k$ for $k=$ $1,2, \ldots, n$. Now it follows as in Henson and Moore [3, Lemma 8.2], that for $j=1,2, \ldots, n$ there is an element $f_{j} \in{ }^{*} X^{\prime}$ with $\left\|f_{j}\right\|<1$ such that $f_{j}\left(p_{i}\right)=\phi_{j}\left(\hat{p}_{i}\right)$ for $i=1,2, \ldots, n$. We thus have $f_{j}\left(p_{i}\right)>r$ for $1 \leqslant j \leqslant i \leqslant n, f_{j}\left(p_{i}\right)=0$ for $1 \leqslant i<j \leqslant n$, and $\left\|T p_{k}\right\|<1 / k$ for $k=1,2, \ldots, n$. It therefore follows by transfer that $T \notin \psi(X, Y)$.

(ii) implies (iii). We check conditions (v) of Theorem 2. First we observe that $\hat{T}\left(\hat{X}_{1}\right)$ is closed in $\hat{Y}$ for any $T \in \mathscr{B}(X, Y)$. Suppose $\hat{T} \hat{p}_{n} \rightarrow \hat{q}$ where $\left\|p_{n}\right\| \leqslant 1$ for $n=1,2, \ldots$ Since $* \mathscr{T} \mathcal{R}$ is assumed to be $\boldsymbol{N}_{1}$-saturated we can extend the sequence of points $\left\{p_{n}: n \in \mathbf{N}\right\}$ to an internal sequence of points in ${ }^{*} X_{1}\left\{p_{n}: n \in{ }^{*} \mathbf{N}\right\}$. If we assume $\left\|T p_{n}-q\right\| \leqslant 1 / n$ for $n \in \mathbf{N}$ it follows that there must be an $\omega \in$ ${ }^{*} \mathbf{N} \backslash \mathbf{N}$ such that $\left\|T p_{\omega}-q\right\| \leqslant 1 / \omega$. This implies $\hat{q}\left(=\hat{T} \hat{p}_{\omega}\right) \in \hat{T}\left(\hat{X}_{1}\right)$. Now suppose (v) b) doesn't hold for $\hat{T}$. Then by the previously used result of James we can assume we have sequences $\left\{\hat{p}_{n}\right\}$ and $\left\{\phi_{n}\right\}$ of norm one elements in $\hat{X}$ and $\hat{X}^{\prime}$ respectively and a real number $r, 0<r<1$ such that for $k=1,2,3, \ldots$ we have $\phi_{n}\left(\hat{p}_{k}\right)>r(n \leqslant k), \phi_{n}\left(\hat{p}_{k}\right)=0(n>k)$ and $\left\|\hat{T}\left(\hat{p}_{k}\right)\right\| \leqslant 1 / k$. This implies $\hat{T} \notin$ $\psi(\hat{X}, \hat{Y})$.

(iii) implies (iv). Suppose $T \notin W \psi(X, Y)$. Then for any (standard) real number $r$ satisfying $0<r<1$ and $\omega \in{ }^{*} \mathbf{N}-\mathbf{N}$ there exist *finite sequences $\left\{p_{1}, p_{2}, \ldots, p_{\omega}\right\}$ and $\left\{f_{1}, f_{2}, \ldots, f_{\omega}\right\}$ of norm one elements in $* X$ and ${ }^{*} X^{\prime}$ respectively such that $f_{j}\left(p_{i}\right)>r$ for $1 \leqslant j \leqslant i \leqslant \omega, f_{j}\left(p_{i}\right)=0$ for $1 \leqslant i<j \leqslant \omega$ and such that $\left\|T p_{k}\right\|<1 / k$ for $k=1,2, \ldots, \omega$. But then the sequences $\left\{\hat{p}_{n}: n \in \mathbf{N}\right\}$ and $\left\{\hat{f}_{n}: n \in \mathbf{N}\right\}$ satisfy $\hat{f}_{j}\left(\hat{p}_{i}\right) \geqslant r$ for $j \leqslant i$ and $\hat{f}_{j}\left(\hat{p}_{i}\right)=0$ for $i<j$ so that as $\left\|\hat{T}_{\hat{p}}\right\| \leqslant 1 / k \hat{T} \notin \widetilde{T}(\hat{X}, \hat{Y})$.

(iv) implies (v). Suppose ker $\hat{T}$ is not superreflexive. Then for all real numbers $r$ such that $0<r<1$ and positive integers $n$ there exist finite sequences of norm 
one elements $\left\{\hat{p}_{1}, \hat{p}_{2}, \ldots, \hat{p}_{n}\right\}$ in $\operatorname{ker} \hat{T}$ and $\left\{\phi_{1}, \phi_{2}, \ldots, \phi_{n}\right\}$ in (ker $\left.\hat{T}\right)^{\prime}$ satisfying $\phi_{j}\left(\hat{p}_{i}\right)>r$ for $1 \leqslant j \leqslant i \leqslant n$ and $\phi_{j}\left(\hat{p}_{i}\right)=0$ for $1 \leqslant i<j \leqslant n$. Extend the functionals $\phi_{1}, \phi_{2}, \ldots, \phi_{n}$ to all of $X$ without increase in norm and, as before, define $f_{1}, f_{2}, \ldots, f_{n} \in{ }^{*} X^{\prime}$ such that $f_{j}\left(p_{i}\right)=\phi_{j}\left(\hat{p}_{i}\right)$ for $i=1,2, \ldots, n$. Consequently for such $r$ and $n$ there are finite sequences of norm one elements $\left\{p_{1}, p_{2}, \ldots, p_{n}\right\}$ in $* X$ and $\left\{f_{1}, f_{2}, \ldots, f_{n}\right\}$ in ${ }^{*} X^{\prime}$ satisfying $f_{j}\left(p_{i}\right)>r$ for $1 \leqslant j \leqslant i \leqslant n$ and $f_{j}\left(p_{i}\right)=0$ for $1 \leqslant i<j \leqslant n$ with $\left\|T p_{k}\right\| \leqslant 1 / k$ for $k=1,2, \ldots, n$. By transfer these elements can be chosen in $X$ and $X^{\prime}$ and consequently $T \notin W \psi(X, Y)$.

Since (v) trivially implies (vi) we are finished once we have shown (vi) implies (i). Suppose $T \notin \psi(X, Y)$. Then for some (standard) $r$ satisfying $0<r<1$ there exist, for $\omega \in{ }^{*} \mathbf{N}-\mathbf{N}$, finite sequence of norm one elements $\left\{p_{1}, p_{2}, \ldots, p_{2 \omega}\right\}$ in $* X$ and $\left\{f_{1}, f_{2}, \ldots, f_{2 \omega}\right\}$ in ${ }^{*} X^{\prime}$ for which $f_{j}\left(p_{i}\right)>r$ for $1 \leqslant j \leqslant i \leqslant 2 \omega, f_{j}\left(p_{i}\right)=0$ for $1 \leqslant i<j \leqslant 2 \omega$ and $\left\|T p_{k}\right\|<1 / k$ for $k=1,2, \ldots, 2 \omega$. For $k=1,2,3, \ldots$ let $\phi_{k}=\hat{f}_{\omega+k}$ and $q_{k}=p_{\omega+k}$. Then the sequence of norm one elements $\hat{q}_{1}, \hat{q}_{2}, \hat{q}_{3}, \ldots$ belongs to $\operatorname{ker} \hat{T}$ and we have $\phi_{j}\left(\hat{q}_{i}\right) \geqslant r$ for $j \leqslant i$ and $\phi_{j}\left(\hat{q}_{i}\right)=0$ for $i<j$. Consequently ker $\hat{T}$ is not reflexive.

In [11] we said an element $T \in \mathscr{B}(X, Y)$ was super weakly compact if for all positive reals $r$ there exists a positive integer $n$ such that there do not exist finite sequences $\left\{x_{1}, x_{2}, \ldots, x_{n}\right\}$ and $\left\{f_{1}, f_{2}, \ldots, f_{n}\right\}$ of norm one elements in $X$ and $Y^{\prime}$ respectively such that $f_{j}\left(T x_{i}\right)>r$ for $1 \leqslant j \leqslant i \leqslant n$ and $f_{j}\left(T x_{j}\right)=0$ for $1 \leqslant i<j$ $\leqslant n$. We were interested in such transformations since $T$ is super weakly compact if and only if $\hat{T} \in \mathcal{U} \mathcal{K}(\hat{X}, \hat{Y})$. Therefore given the equivalence of (i) and (iii) and the truth of the analogous results for $\mathscr{T}(X, Y)$ we can note:

(1) $\psi(X, Y)$ is closed under perturbations by super weakly compact transformations from $X$ into $Y$.

(2) If $T \in \mathscr{B}(X, Y), S \in \mathscr{B}(Y, Z)$ and $S T \in \psi(X, Z)$ then $T \in \psi(X, Y)$.

(3) $\psi(X, Y)$ equals $\mathscr{B}(X, Y)$ if $X$ is superreflexive. If $X$ is non-superreflexive then $\psi(X, Y)$ doesn't contain any super weakly compact transformations, and so is empty if $Y$ is superreflexive.

Our final result that $\psi(X, Y)$ is open depends upon the observation that the proof of (ii) implies (iv) given in Theorem 3 does not depend on $T$ being standard. That is to say if $S \in$ finite $* \mathscr{\Re}(X, Y)$ and $\hat{S} \in \psi(\hat{X}, \hat{Y})$ then $S \in{ }^{*} W \psi(X, Y)=$ ${ }^{*} \psi(X, Y)$. A direct proof proceeds as follows. Suppose $S \notin * \psi(X, Y)$ so that $S \#^{*} W \psi(X, Y)$. Then for each (standard) $r$ satisfying $0<r<1$ and (standard) positive integer $n$ there exist finite sequences of norm one elements $\left\{p_{1}, p_{2}, \ldots, p_{n}\right\}$ in $* X$ and $\left\{f_{1}, f_{2}, \ldots, f_{n}\right\}$ in $* X^{\prime}$ for which $f_{j}\left(p_{i}\right)>r$ for $1 \leqslant j \leqslant i \leqslant n, f_{j}\left(p_{i}\right)=0$ for $1 \leqslant j<i \leqslant n$, and $\left\|S p_{k}\right\|<1 / k$ for $k=1,2, \ldots, n$. But then $\hat{f}_{j}\left(\hat{p}_{i}\right) \geqslant r$ for $1 \leqslant j \leqslant i \leqslant n, \hat{f}_{j}\left(\hat{p}_{i}\right)=0$ for $1 \leqslant i<j \leqslant n$ and $\left\|\hat{S} \hat{p}_{k}\right\| \leqslant 1 / k$ for $k=1,2, \ldots, n$. This implies $\hat{S} \notin \psi(\hat{X}, \hat{Y})$. 
Theorem 4. Let $X$ and $Y$ be Banach spaces. Then the class $\psi(X, Y)$ forms an open subset of $\mathscr{G}(X, Y)$.

Proof. Suppose $T \in \psi(X, Y)$ and consider a transformation $S \in *$ S $(X, Y)$ with infinitesimal norm. Then $(T+S) \hat{T} \in \psi(\hat{X}, \hat{Y})$ by Theorem 3 , and so $T+S \in{ }^{*} \psi(X, Y)$ by the above remark. Thus the set $\left\{\varepsilon>0 \mid \forall S \in *^{*}(X, Y)\right.$, $\|S\|<\varepsilon$ implies $\left.T+S \in{ }^{*} \psi(X, Y)\right\}$ is an internal set containing the positive infinitesimals. Hence it contains a standard positive $\varepsilon$, and we consequently have our result.

This result shows that at least from this point of view $\psi(X, Y)$ has an analogous role to that of $\phi_{+}(X, Y)$. Given this it is natural to define a class $\psi(X, Y)$ of generalized Fredholm transformations by the property that

$$
T \in \psi(X, Y) \text { if and only if } T \in \psi(X, Y) \text { and } T^{\prime} \in \psi\left(Y^{\prime}, X^{\prime}\right) .
$$

Such a class is then open and is closed under perturbation by super weakly compact transformations. There are of course other ways of generalizing Fredholm transformations. Yang ([12], [13]) has done this in two ways; namely he has considered transformations with closed range and reflexive kernel and cokernel, and transformations invertible modulo the weakly compact transformations. These transformations in general do not share the stability properties of our $\psi(X, Y)$ but the three classes are closely related.

\section{Acknowledgement}

The author thanks the referee for providing him with the references to the two papers of Yang. It was through these papers that the author became aware of the work of Kalton and Wilansky on Tauberian transformations. This lead to a simplification of the initial paper.

\section{References}

1. S. R. Caradus, W. E. Pfaffenberger and Bertram Yood, Calkin algebras and algebras of operators on Bunach spaces (Lecture Notes in Pure and Applied Mathematics, Dekker, New York, 1974).

2. J. J. M. Chadwick and A. W. Wickstead, 'A quotient of ultrapowers of Banach spaces and semi-Fredholm operators', Bull. London Math. Soc. 9 (1977), 321-325.

3. C. W. Henson and L. C. Moore, Jr., 'The nonstandard theory of topological vector spaces', Trans. Amer. Math. Soc. 172 (1972), 405-435, Erratum, ibid 184 (1973), 509.

4. R. C. James, 'Weakly compact sets', Trans. Amer. Math. Soc. 113 (1964), 129-140.

5. R. C. James. 'Some self-dual properties of normed linear spaces'. Sympos. on Infinite Dimensional Topologr, pp. 159-175 (Ann. of Math. Studies 69. Princeton Univ. Press. Princeton. 1972). 
6. N. Kalton and A. Wilansky, 'Tauberian operators on Banach spaces', Proc. Amer. Math. Soc. $57(1976), 251-255$.

7. A. Lebow and M. Schechter, 'Semigroups of operators and measures of noncompactness', $J$. Functional Analysis 7 (1971), 1-26.

8. W. A. J. Luxemburg, 'A general theory of monads', Application of Model Theory to Algebra, Analysis and Probability, Internat. Sympos., Pasadena, Calif., 1967, pp. 18-86, (Holt, Rinehart and Winston, New York, 1969).

9. W. A. J. Luxemburg, 'On some concurrent binary relations occurring in analysis', Contributions to nonstandard analysis, pp. 85-100 (Studies in Logic and the Foundations of Math., NorthHolland, Amsterdam, London, 1972).

10. A. Robinson, Non-standard analysis (Studies in Logic and the Foundations of Math., NorthHolland, Amsterdam, 1966).

11. D. G. Tacon, 'Nonstandard extensions of transformations between Banach spaces', Trans. Amer. Math. Soc. 260 (1980), 147-158.

12. K. W. Yang, 'The generalized Fredholm operators', Trans. Amer. Math. Soc. 216 (1976), 313-326.

13. K. W. Yang, 'Operators invertible modulo the weakly compact operators', Pacific J. Math. 71 (1977), 559-564.

\author{
University of New South Wales \\ Box 1, Post Office \\ Kensington, N.S.W., 2033 \\ Australia
}

